\title{
Real-time spectral imaging in three spatial dimensions
}

\author{
Wenhai Liu* and Demetri Psaltis \\ Department of Electrical Engineering, California Institute of Technology, Mail Stop 136-93, Pasadena, California 91125
}

George Barbastathis

Department of Mechanical Engineering, Massachusetts Institute of Technology, Room 3-461c, 77 Massachusetts Avenue, Cambridge, Massachusetts 02139

Received December 17, 2001

We report what is to our knowledge the first volume-holographic optical imaging instrument with the capability to return three-dimensional spatial as well as spectral information about semitranslucent microscopic objects in a single measurement. The four-dimensional volume-holographic microscope is characterized theoretically and experimentally by use of fluorescent microspheres as objects. @ 2002 Optical Society of America OCIS codes: $090.2890,110.0110,090.1970,090.7330,100.6890$.

Classical imaging systems process the optical field by use of elements such as lenses, apertures and stops, and thin diffraction gratings. By placing several such elements in tandem, one can capture projections of very general objects, e.g., containing three-dimensional (3D) spatial as well as spectral information. We refer to such objects as four dimensional (4D). The projections that these systems are capable of forming are twodimensional (2D) or lower; scanning is needed to span the entire $3 \mathrm{D}$ or $4 \mathrm{D}$ object space.

For example, a classical confocal microscope ${ }^{1-3}$ uses a combination of objective-collector lenses and a pinhole to capture information about a single point in the object and acquires a zero-dimensional projection at every measurement. Scanning along three dimensions is needed to acquire the 3D spatial structure of the object. By providing spectral scanning means (e.g., a monochromator or a scanning Fabry-Perot interferometer), one can also acquire spectral information, albeit in a very time-consuming procedure. Optical coherence tomography ${ }^{4}$ requires only 3D scanning for capturing spatial information, whereas spectral information is recovered digitally from the phase of the correlation function of the optical beam. ${ }^{5}$ Coherence imaging ${ }^{6-9}$ returns $2 \mathrm{D}$ projections in the Fourier (k) space at the expense of dynamic range. Here we report what is, to our knowledge, the first instrument with the capability to acquire spatial and spectral information simultaneously (in a single measurement). Therefore, real-time 4D imaging becomes possible at rates specified by the photon count and not the scanning speed.

The $4 \mathrm{D}$ imaging capability is based on the Bragg diffraction selectivity and degeneracy properties of volume holograms. ${ }^{10-12}$ The principle of volume-holographic imaging is illustrated in Fig. 1. The optical field emitted or scattered by a 4D object is transformed by the appropriate combination of lenses and subsequently diffracted by a volume-holographic optical element, which has been prerecorded to multiple superimposed holograms. Each hologram is tuned to its corresponding $2 \mathrm{D}$ slice of the $4 \mathrm{D}$ object. If the projected slices span the entire $4 \mathrm{D}$ object space, then the need for scanning is eliminated.

0146-9592/02/100854-03\$15.00/0
In this Letter we discuss and experimentally characterize a specific holographic imaging of transmission geometry. With the detailed structure shown in Fig. 2, the hologram is recorded by interfering the signal beam, collimated from a monochromatic coherent point source at $\left(x_{r}, y_{r}, z_{r}, \lambda_{r}\right)$ and its coherent plane reference beam in the $-\hat{x}$ direction. An imaging lens focuses the diffracted beam from the volume-holographic optical element onto a 2D detector array surface. For simplicity we consider the simplest possible object: a probe point source of arbitrary wavelength $\lambda_{p}$, located in the vicinity of the recording point source.

When the probe is displaced by $\Delta x_{p}\left(\left|\Delta x_{p}\right| \ll f_{c}\right)$ in the $\hat{x}$ direction, the collimated signal beam rotates in the $x z$ plane. According to the well-known angle selectivity, the diffraction efficiency, $\eta\left(\Delta x_{p}\right)=$ $\operatorname{sinc}^{2}\left(\Delta x_{p} / f_{c} \Delta \theta\right)$, drops to zero (the first null) at $\Delta \theta_{S}$ :

$$
\Delta \theta_{S}=\frac{\left|\Delta x_{p}\right|}{f_{c}}=\frac{\lambda}{D} \frac{1}{\cos \theta_{S}\left(\tan \theta_{S n}+\tan \theta_{R n}\right)},
$$

where $D$ is the thickness of the hologram and $\theta_{\mathrm{S} n}, \theta_{\mathrm{R} n}$, and $\theta_{S}$ are the incident angles inside or outside the holographic material in Fig. 2. If instead the probe point source is displaced relative to the recording point source by $\Delta z_{p}$ in depth, the light after the collimator lens is a spherical wave. The diffraction efficiency can be approximated to first order by incoherent addition of all spatial frequency components of the defocused beam in the $x z$ plane $^{10}$ as

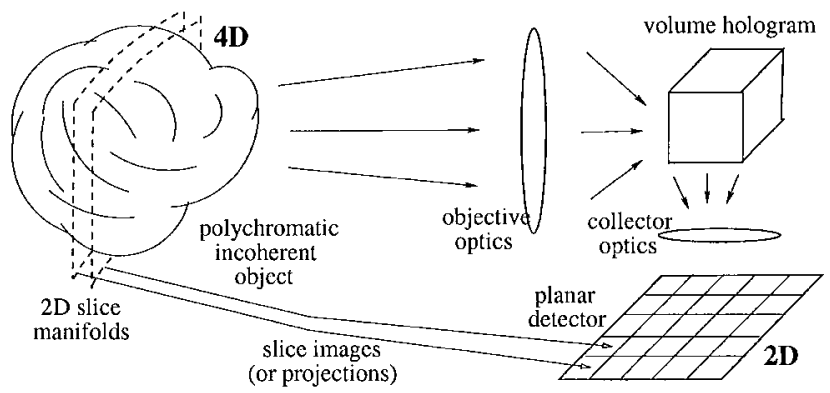

Fig. 1. Volume-holographic 4D imaging principle. 


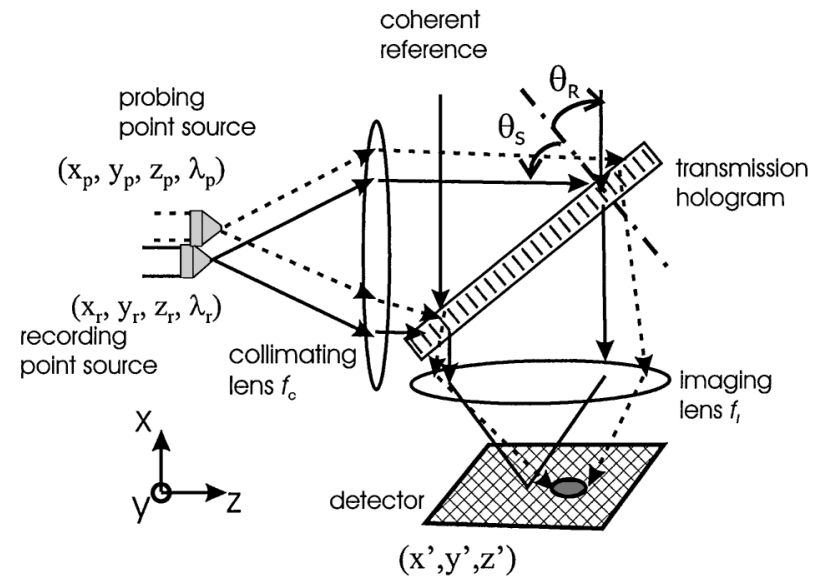

Fig. 2. Experimental recording and imaging geometry.

$$
\eta\left(\Delta z_{p}\right)=\frac{1}{\alpha} \int_{0}^{\alpha} \operatorname{sinc}^{2}\left(\frac{t}{\Delta \theta_{S}}\right) \mathrm{d} t \approx \frac{\Delta \theta_{S}}{2 \pi \alpha} \operatorname{Si}\left(\frac{2 \pi \alpha}{\Delta \theta_{S}}\right)
$$

where $\operatorname{Si}(s) \equiv \int_{0}^{s}(\sin t / t) \mathrm{d} t, \quad \alpha=L \Delta z_{p} / 2 f_{c}{ }^{2}$, and $L$ is the collimating lens aperture. Finally, for a small probe wavelength deviation $\left|\Delta \lambda_{p}\right| \equiv \mid \lambda_{p}-$ $\lambda_{r} \mid<<\lambda_{r}$, the diffraction efficiency drops, to first order, as $\eta\left(\Delta \lambda_{p}\right)=\operatorname{sinc}^{2}\left(\Delta \lambda_{p} / \lambda_{r} \Delta \beta\right)$, where

$$
\Delta \beta=\frac{\lambda_{r}}{n D} \frac{\cos \theta_{R n}}{1-\cos \left(\theta_{S n}+\theta_{R n}\right)} .
$$

The experimental and theoretical spatial selectivities are compared in Fig. 3 and have good agreement. These diffraction efficiencies give the imaging resolution on $\Delta x_{p}, \Delta z_{p}$, and $\Delta \lambda_{p}$, which are determined by the hologram thickness, $D$, and the objective lens, $f_{c}$.

Two basic Bragg degeneracies (i.e., Bragg matching with a probing source that is different from the recording source ${ }^{13}$ ) exist in the transmission geometry. Consider a single grating $\mathbf{K}$ recorded by the reference-signal wave-vector pair $\left(\mathbf{k}_{\mathrm{R} r}, \mathbf{k}_{\mathrm{S} r}\right), k_{r}=2 \pi / \lambda_{r}$, all probe-diffracted wave-vector pairs $\left(\mathbf{k}_{\mathrm{R} p}, \mathbf{k}_{\mathrm{S} p}\right)$ are Bragg matched when $\mathbf{K}=\mathbf{k}_{\mathrm{Rp}}-\mathbf{k}_{\mathrm{S} p}$. The first degeneracy is $\left(\mathbf{k}_{\mathrm{R} p}, \mathbf{k}_{\mathrm{S} p}\right)$ at wavelength $\lambda_{p}=\lambda_{r}$, obtained by rotation of $\left(\mathbf{k}_{\mathrm{R} p}, \mathbf{k}_{\mathrm{S} r}\right)$ about K. ${ }^{13}$ In Fig. 2 , this degeneracy means the probe source moving along $\Delta y_{p}$ and the imaging point along $\Delta y_{i}=\Delta y_{p}\left(f_{i} / f_{p}\right)$. The second degeneracy is for pairs $\left(\mathbf{k}_{\mathrm{R} p}, \mathbf{k}_{\mathrm{S} p}\right)$ at wavelengths $\lambda_{p} \neq \lambda_{r}{ }^{10,14}$ This corresponds to the probe source along a spatial-spectral coupled direction in Fig. 2, satisfying

$$
\frac{\Delta x_{p}}{f_{c}}=-\frac{\Delta \lambda_{p}}{\lambda_{r}}
$$

and yielding an image at the output plane with $\Delta z^{\prime}=$ $\left(f_{i} / f_{c}\right) \Delta x_{p}$ relative to the location of the reference image point.

All object (or probe) point sources along the two Bragg degenerate dimensions are reconstructed onto the image $y^{\prime}-z^{\prime}$ plane. Therefore, a 2D slice at a fixed depth in a $y_{p}-\left(x_{p} / \lambda_{p}\right)$ spatial-spectral coupled plane out of the $4 \mathrm{D}$ object space can be extracted by a single volume hologram. The experimental demonstration of optical sectioning and imaging is quantified

(a)

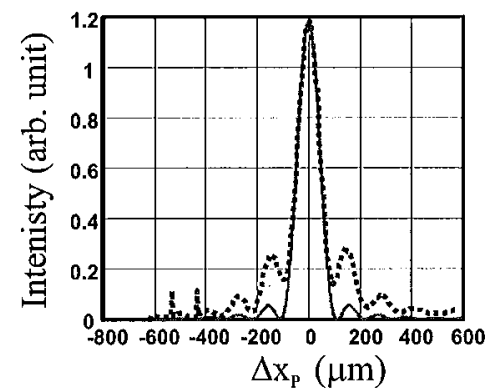

(b)

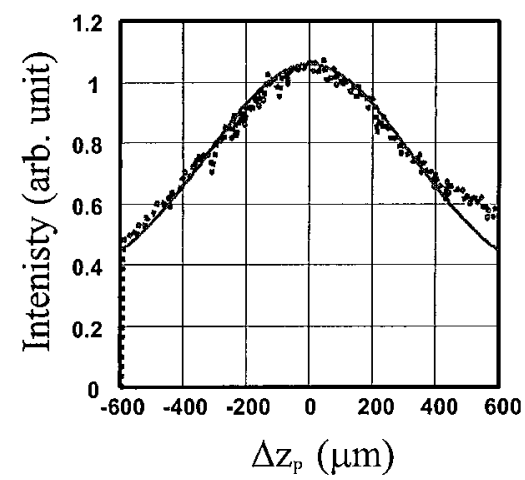

Fig. 3. Diffraction efficiency of theoretical calculation (solid curves) and experimental measurement (dotted curves) as a function (a) of $\Delta x_{p}$ and (b) $\Delta z_{p}$ in Fig. 2. The hologram was recorded and probed with an $\mathrm{Ar}^{+}$ laser at $488 \mathrm{~nm}$, with a $10 \times, 0.25$-N.A. objective lens as the collimating lens, in holographic Dupont HRF-150 photopolymer $(D=100 \mu \mathrm{m}, n \approx 1.5)$, leading to resolution $\Delta x_{p}=104 \mu \mathrm{m}$ (first null) and $\Delta z_{p}=400 \mu \mathrm{m}$ (FWHM).

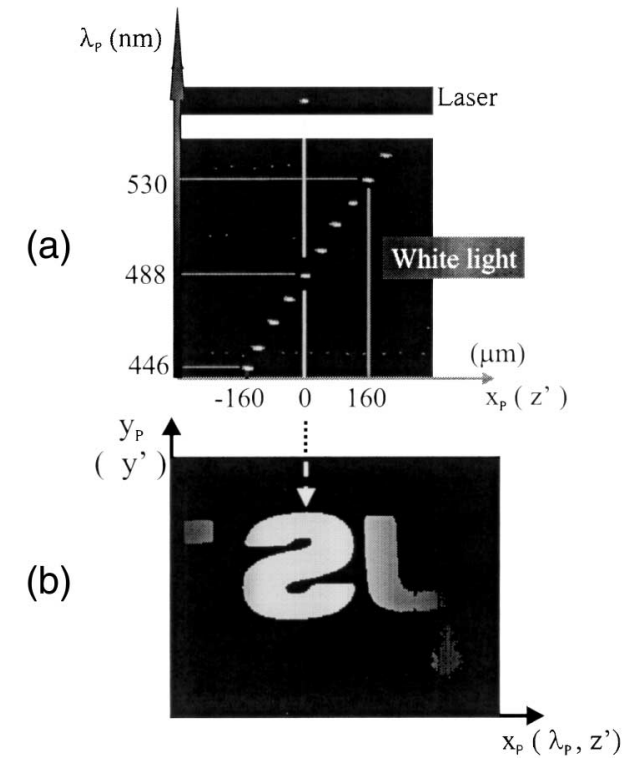

Fig. 4. Observation of Bragg degeneracies in Fig. 2, with a $10 \times, 0.25-$ N.A. objective lens in a holographic material, $\mathrm{LiNbO}_{3}(D=5 \mathrm{~mm})$. (a) Response to a point source object of varying wavelength and moving along lateral $\hat{x}$ position. (b) Response to a mask (2D object) illuminated with white light. 
(a)

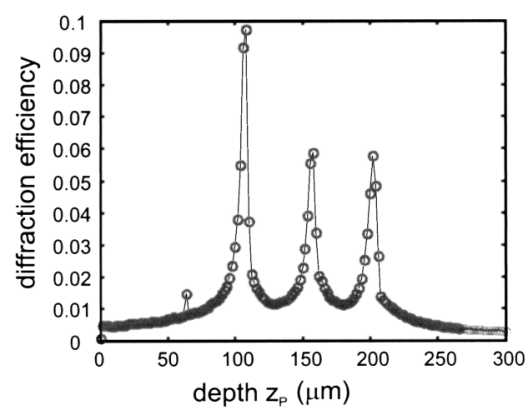

(b)

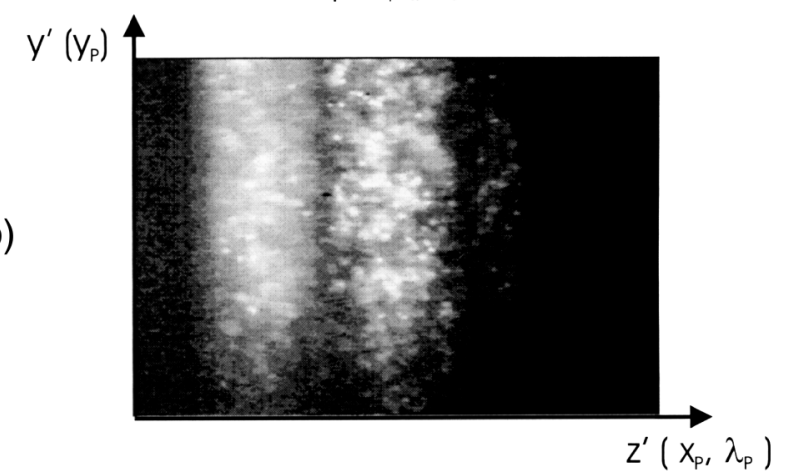

Fig. 5. Simultaneous optical sectioning by use of multiplexed holograms. Three holograms were recorded at $488 \mathrm{~nm}$ (see Fig. 2) with a $40 \times, 0.65-$ N.A. objective lens in a holographic material, phenanthrequinoneembedded PMMA ( $D=2 \mathrm{~mm}, n \approx 1.5)$. Three recording point sources were on axis and separated by $50 \mu \mathrm{m}$ in the longitudinal (depth) direction. Each hologram had a spatial-spectral resolution of $\Delta z_{p}=3 \mu \mathrm{m}$ (FWHM), $\Delta x_{p}=1 \mu \mathrm{m}$ (first null), and $\Delta \lambda_{p}=0.16 \mathrm{~nm}$. (a) Response to monochromatic probing point source $\left(\lambda_{r}=488 \mathrm{~nm}\right)$ along depth. (b) Simultaneous imaging of three slices from a liquid sample containing fluorescent microspheres (Molecular Probes F-8844 polystyrene microsphere, fluorescent yellow-green 505-515-nm, diameter $15 \mu \mathrm{m}$, pumped at $488 \mathrm{~nm}$ ).

in Fig. 4. When the single hologram was probed with a monochromatic point source (a 5 - $\mu \mathrm{m}$ pinhole under a 488-nm $\mathrm{Ar}^{+}$laser) along the $\hat{x}$ direction at the entrance focal plane, significant diffraction was obtained at only one location (the recording pinhole location at $488 \mathrm{~nm}$ ). However, when the probe pinhole was illuminated by a white-light source, the image point location shifted along $\hat{z^{\prime}}$, while the wavelength changed in response to Eq. (4), as shown in Fig. 4(a). Both degeneracies in action simultaneously led to the horizontal-dependent rainbow (spectral) image in Fig. 4(b), when the object was a 2D mask illuminated by the white-light source.

Multiple 2D sections can be extracted simultaneously from a $4 \mathrm{D}$ object if a volume-holographic optical element with multiplexed holograms is used instead. Each hologram is arranged to extract a corresponding slice and to diffract the light from that slice toward a nonoverlapping section of the detector. Figure 5 shows three corresponding slices along the $\hat{z}$ direction of the object that were acquired in a single measurement from a liquid sample containing fluorescent microspheres. The slices were obtained by use of three multiplexed holograms. Each hologram reconstructs a single layer of the microspheres inside the sample. The width of the visible bands is determined by the fluorescence bandwidth according to Eq. (2).

In conclusion, we have demonstrated and quantified a volume-holographic imaging instrument with real-time $4 \mathrm{D}$ imaging capability. The $4 \mathrm{D}$ imaging resolution values $\Delta x_{p}, \Delta z_{p}$, and $\Delta \lambda_{p}$ are related to one another by the hologram geometry parameters. Higher resolution requires greater illumination for a photon imaging rate; e.g., $\Delta \lambda_{p}=0.16 \mathrm{~nm}$ in Fig. 5 is too small, and the photon efficiency is sacrificed to keep spatial resolution. Hologram multiplexing also reduces the diffraction efficiency. Detailed design and resolution-efficiency trade-off depend on the practical application requirements.

We are grateful to Arnab Sinha and Michal Balberg for valuable discussions and to the following sponsors: the National Science Foundation, the Defense Advanced Research Projects Agency, and the U.S. Air Force Research Laboratories. The authors' e-mail addresses are wliu@sunoptics.caltech.edu, psaltis@sunoptics.edu, and gbarb@mit.edu.

* Present address, Ondax, Inc., 850 East Duarte Road, Monrovia, California 91016.

\section{References}

1. M. Minsky, "Microscopy apparatus," U.S. patent 3,013,467 (December 19, 1961).

2. T. Wilson, Confocal Microscopy (Academic, San Diego, Calif., 1990).

3. J. K. Stevens, L. R. Mills, and J. E. Trogadis, eds., Three-Dimensional Confocal Microscopy: Volume Investigation of Biological Systems (Academic, San Diego, Calif., 1994).

4. D. Huang, E. A. Swanson, C. P. Lin, J. S. Schuman, W. G. Stinson, W. Chang, M. R. Hen, T. Flotte, K. Gregory, C. A. Puliafito, and J. G. Fujimoto, Science 254, 1178 (1991).

5. U. Morgner, W. Drexler, F. X. Kartner, X. D. Li, C. Pitris, E. P. Ippen, and J. G. Fujimoto, Opt. Lett. 25, 111 (2000).

6. W. H. Carter and E. Wolf, Opt. Acta 28, 227 (1981).

7. K. Itoh and Y. Ohtsuka, J. Opt. Soc. Am. A 3, 94 (1986).

8. J. Rosen and A. Yariv, J. Opt. Soc. Am. A 13, 2091 (1996).

9. D. L. Marks, R. A. Stack, D. J. Brady, D. C. Munson, Jr., and R. B. Brady, Science 284, 2164 (1999).

10. G. Barbastathis and D. Psaltis, Opt. Lett. 21, 429 (1996).

11. G. Barbastathis, M. Balberg, and D. J. Brady, Opt. Lett. 24, 811 (1999).

12. G. Barbastathis and D. J. Brady, Proc. IEEE 87, 2098 (1999).

13. H. Lee, X.-G. Gu, and D. Psaltis, J. Appl. Phys. 65, 2191 (1989).

14. D. Psaltis, F. Mok, and H. Y.-S. Li, Opt. Lett. 19, 210 (1994). 